\title{
ESPOROTRICOSIS EN PACIENTES QUE ACUDEN A UN CENTRO MÉDICO DE REFERENCIA EN ABANCAY, PERÚ
}

\author{
Max Carlos Ramírez-Soto1,a, Jonathan Andagua-Castro1,a, José Lizárraga-Trujilloº, \\ Elsa Gladys Aguilar-Ancori'1,a, Justo Darwin Pezo-Ochoa ${ }^{3, a}$
}

\begin{abstract}
RESUMEN
Se realizó un estudio descriptivo entre mayo y agosto de 2008 en el Centro Médico Santa Teresa de la ciudad de Abancay, departamento de Apurimac, Perú. Se incluyó a 33 pacientes con sospecha clínica de esporotricosis; se identificó por cultivo la presencia del Sporothrix schenckii en 20 pacientes. Once casos (55\%) fueron de sexo masculino, mientras que el grupo más afectado fue el de niños de 0 a 14 años. El $60 \%$ de las lesiones fueron cutánea fijas ubicadas con mayor frecuencia en la cabeza y cuello (40 \%). El $55 \%$ tuvo un tiempo de evolución menor o igual a un mes. La presentación de esporotricosis en población pediátrica, con compromiso de cabeza y cuello, debe considerarse en el diagnóstico diferencial de lesiones cutáneas de pacientes provenientes de zonas endémicas.
\end{abstract}

Palabras clave: Esporotricosis; Sporothrix schenckii; Abancay (fuente: DeCS BIREME).

\section{SPOROTRICHOSIS IN PATIENTS ATTENDING A REFERENCE CENTER IN ABANCAY, PERU}

\begin{abstract}
A descriptive study was conducted between the months of May and August 2008 in Santa Teresa Medical Center, Abancay. Of 33 patients clinically suspected with Sporotrichosis: 20 patients were identified with Sporothrix schenckii. $11(55 \%)$ of patients were males. Children between 0 and 14 years were most affected. $60 \%$ of the lesions were fixed cutaneous being more frequent in the head and neck (40\%). 55\% had lessions for less than one month. In pediatric population with involvement of head and neck, Sporotrichosis should be considered in the differential diagnosis of skin lesions of patients from endemic areas.
\end{abstract}

Key words: Sporotrichosis; Sporothrix schenckii; Abancay (source: MesH NLM).

\section{INTRODUCCIÓN}

La esporotricosis es una infección fúngica subcutánea ocasionada por el hongo dimórfico Sporothrix schenckii. El hábitat y las fuentes potenciales de infección están relacionados con plantas espinosas, materia orgánica en descomposición, tierras de alfarería (1) y, en otros casos, rasguño o mordedura de gatos ${ }^{(2)}$. Su desarrollo y diseminación es favorecido por las condiciones del lugar: viviendas construidas informalmente, casas de adobe, pisos de tierra y condiciones deficientes de higiene ${ }^{(3)}$. El hongo ingresa por inoculación traumática y asciende por los canales linfáticos para luego desarrollar lesiones granulomatosas y generar una pápula ulcerativa nodular, que es la presentación clínica más frecuente; también puede presentarse en forma cutánea fija, cutánea diseminada o sistémica ${ }^{(4)}$. Afecta a individuos de ambos sexos y de cualquier edad (1). El estado inmunológico del huésped, el sitio de inoculación y la virulencia del hongo son determinantes en la producción de estas manifestaciones clínicas ${ }^{(5,6)}$.

La distribución de esta micosis es amplia, con diversos focos endémicos alrededor del mundo; en Latinoamérica la encontramos en Brasil, Colombia, México, Centroamérica, Perú, Venezuela y Uruguay. Los casos notificados en el ámbito nacional provienen de los departamentos de La Libertad, Ayacucho, Apurímac y Cusco ${ }^{(7)}$. La provincia de Abancay, capital de Apurímac, es una zona hiperendémica de esporotricosis debido a la alta incidencia anual que presenta (98 casos por cada 100000 habitantes) y donde la infección se asocia con las precarias condiciones de las viviendas $y$, sobre todo, al factor socioeconómico ${ }^{(8)}$; afecta más a los niños y es

\footnotetext{
Facultad de Ciencias Biológicas, Universidad Nacional de San Antonio Abad del Cusco. Cusco, Perú.

2 Gobierno Regional Apurímac. Apurímac, Perú.

3 Laboratorio Referencial, Dirección Regional de Salud Apurímac. Apurímac, Perú.

a Biólogo; ${ }^{b}$ Médico Dermatólogo
}

Recibido: 12-05-11 Aprobado: 10-08-11 
considerada como una micosis significativa de las áreas rurales del Perú ${ }^{(9)}$.

El Centro Médico Santa Teresa (CMST) es el centro referencial de diagnóstico y tratamiento de esporotricosis en la ciudad de Abancay, en este lugar se atiende a pacientes con sospecha de la infección y es también donde se reportan el mayor número de casos. El objetivo del estudio fue identificar la positividad del cultivo en casos con sospecha clínica de esporotricosis y evaluar las características de la población afectada.

\section{EL ESTUDIO}

Se realizó un estudio descriptivo durante los meses de mayo a agosto del año 2008 en el CMST de Abancay, ciudad ubicada a $2378 \mathrm{~m}$ de altitud.

Aspectos éticos. El estudio fue aprobado por la Facultad de Ciencias Biológicas de la Universidad Nacional de San Antonio Abad del Cusco. Cada paciente fue informado brevemente acerca de la biología del hongo y de las características de la enfermedad; también se detalló los objetivos y beneficios del estudio, y se les entregó una hoja de consentimiento informado, en el caso de los menores de 18 años el consentimiento informado fue firmado por sus padres. La publicación de las fotografías fue autorizada por los pacientes.

Recolección de datos. Se realizaron encuestas en una ficha epidemiológica la cual contenía datos demográficos del paciente, antecedentes, factores asociados y aspectos clínicos de la enfermedad.

Diagnóstico de esporotricosis. Se realizó el cultivo micológico en agar Sabouraud, considerando positivo el crecimiento del Sporothrix schenckii. Los cultivos fueron leídos hasta los 30 días, periodo luego del cual fueron considerados como negativos en caso de no evidenciarse crecimiento. Todos los cultivos fueron confirmados por el Laboratorio de Micología del Centro Nacional de Salud Pública del Instituto Nacional de Salud.

Análisis de datos. Para el análisis estadístico se elaboró una base de datos en el programa Excel 97 donde se realizaron comparaciones estadísticas entre el sexo, edad, ocupación, tipo de lesión, tiempo y localización de las lesiones en pacientes en los cuales se aisló el agente etiológico.

\section{HALLAZGOS}

Durante el periodo de estudio se incluyeron 33 casos de pacientes con diagnóstico clínico de esporotricosis.
Tabla 1. Distribución de pacientes con esporotricosis según edad, sexo, tipo de lesión, localización, tiempo de lesión y ocupación.

\begin{tabular}{|c|c|c|}
\hline \multirow[t]{2}{*}{ Variables } & \multicolumn{2}{|c|}{ Total } \\
\hline & $(n=20)$ & $(\%)$ \\
\hline \multicolumn{3}{|l|}{ Sexo } \\
\hline Masculino & 11 & $(55)$ \\
\hline Femenino & 9 & $(45)$ \\
\hline \multicolumn{3}{|l|}{ Edad (años) } \\
\hline $0-14$ & 9 & $(45)$ \\
\hline $15-45$ & 8 & $(40)$ \\
\hline$>45$ & 3 & (15) \\
\hline \multicolumn{3}{|l|}{ Tipo de lesión } \\
\hline Cutánea Fija & 12 & $(60)$ \\
\hline Cutánea Linfática & 8 & $(40)$ \\
\hline \multicolumn{3}{|l|}{ Localización } \\
\hline Cabeza y cuello & 8 & $(40)$ \\
\hline Miembros superiores & 7 & (35) \\
\hline Otros & 5 & $(25)$ \\
\hline \multicolumn{3}{|l|}{ Tiempo de lesión } \\
\hline$\leq 1 \mathrm{mes}$ & 11 & $(55)$ \\
\hline$>1$ mes & 9 & $(45)$ \\
\hline \multicolumn{3}{|l|}{ Ocupación } \\
\hline Estudiante & 11 & $(55)$ \\
\hline Ama de casa & 2 & (10) \\
\hline Agricultor & 2 & (10) \\
\hline Otros & 5 & $(25)$ \\
\hline
\end{tabular}

Veinte de ellos tuvieron confirmación por cultivo y fueron incluidos en el análisis final. Once casos fueron de sexo masculino y las proporciones de pacientes con lesiones con tiempo de evolución menor o mayor a un mes fueron similares. Se encontró 19 casos con lesiones solo en una región anatómica y uno en dos localizaciones distintas. Las características de la población atendida se muestran en la Tabla 1. Las Figuras 1, 2 y 3 muestran las características típicas de las lesiones.

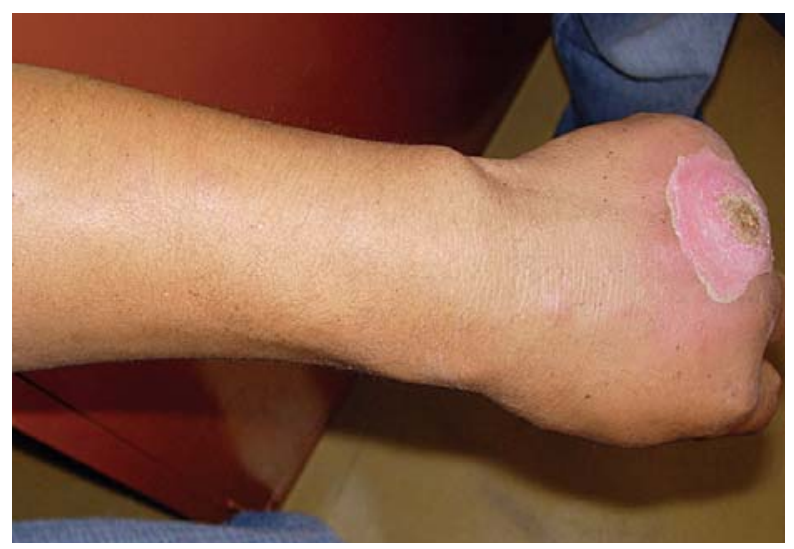

Figura 1. Esporotricosis cutánea linfática. Lesión ulcerativa eritematosa maculopapular en el dorso de la mano con presencia de nódulos a lo largo del brazo. 


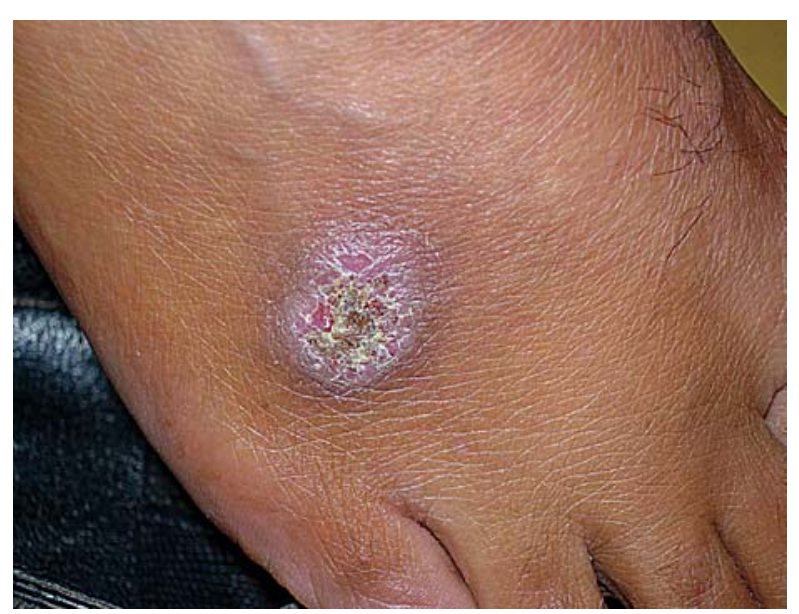

Figura 2. Esporotricosis cutánea fija. Placa eritematoviolácea escamocostrosa en miembro inferior.

\section{DISCUSIÓN}

La alta frecuencia de esporotricosis en Abancay, probablemente se deba a las condiciones climáticas favorables para el crecimiento y desarrollo del hongo. Otras zonas andinas como Cusco, también han sido reportadas como zonas endémicas de la infección ${ }^{(7)}$. Esto contrasta con la mayoría de publicaciones, que describen a esta enfermedad en zonas de climas tropicales y subtropicales ${ }^{(1,10-12)}$.

Clásicamente, se ha considerado a la esporotricosis una enfermedad ocupacional relacionada directamente con actividades propias del campo que incluyen manipulación de la vegetación y contacto con el suelo ${ }^{(13)}$. En contraste, diversos estudios la consideran como una enfermedad predominantemente pediátrica ${ }^{(2,7,8,14)}$. El presente estudio encontró que el grupo etario más afectado

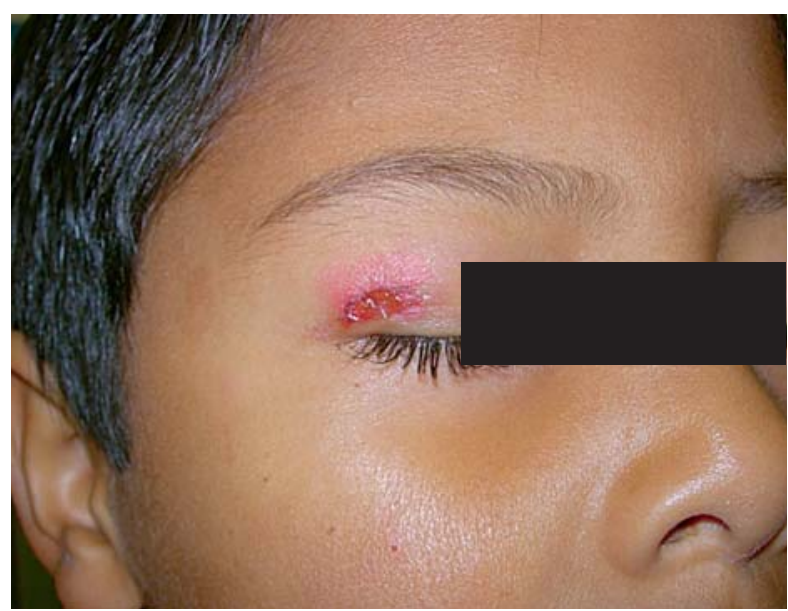

Figura 3. Esporotricosis cutánea fija. Lesión ulcerativa en el párpado superior derecho. fue el de 0 a 14 años, en el cual se encontró, incluso, niños menores de 5 años. De acuerdo con publicaciones previas basadas en población nacional, alrededor de dos terceras partes de casos pueden presentarse en población pediátrica ${ }^{(8,9)}$. Una probable explicación es que los niños provenían de zonas urbano - rurales, donde están más expuestos al agente patógeno y realizan actividades agrícolas desde temprana edad, como manipular vegetales, tierra o instrumentos punzocortantes, lo cual genera una puerta de ingreso al hongo ${ }^{(15)}$. Otro factor de riesgo descrito ha sido el contacto con gatos, los que aparentemente pueden actuar como reservorios de la enfermedad, (11,12).

En cuanto al sexo, el $55 \%$ de casos fueron de sexo masculino. La literatura revisada muestra resultados discordantes en cuanto a la distribución, por sexo, de la enfermedad $^{(2,7,9,13)}$.

De acuerdo con la mayoría de autores, la forma de lesión cutánea-linfática comprende alrededor del $75 \%$ de todos los casos estudiados ${ }^{(1,7,15)}$. Sin embargo, nuestros resultados son similares a los estudios anteriormente realizados en el CMST, donde la mayoría de casos fueron lesiones cutánea fijas ${ }^{(2)}$, y a otros reportes de Abancay ${ }^{(16)}$. Es pertinente considerar que en otros estudios en Abancay, la lesión cutánea-linfática fue la más frecuente ${ }^{(8)}$. Estas diferencias con nuestros resultados posiblemente se debe a que la lesión cutánea-fija es más frecuente en lugares endémicos y presenta diversas formas clínicas (verrucosa, ulcerativa, eritematosa, etc.) ${ }^{(4)}$.

En contraste a la predominancia clásica de lesiones en las extremidades, se ha descrito que en áreas endémicas las lesiones en la cara afectan más a los niños $(13,14)$. En este estudio encontramos que la ubicación más frecuente fue en la cabeza y en el cuello (40\%), que incluía casos de esporotricosis palpebral en niños que, probablemente, se infectaron por jugar con tierra, por falta de higiene o el rascarse con las uñas cargadas de tierra contaminada en zonas de piel delicada, la cual actúa como un factor favorable para la instalación del hongo ${ }^{(14)}$. Los resultados presentados no muestran diferencias con los estudios de Abancay los que también encontraron lesiones frecuentes en la cara y cuello de niños ${ }^{(3,8,9)}$. En miembros superiores se presentó en un $30 \%$, debido a que estas regiones se encuentran descubiertas y con mayor exposición al medioambiente, lo cual, debido al traumatismo provocado por rascarse, facilita la entrada del hongo ${ }^{(7,17)}$; los miembros inferiores y otras regiones anatómicas fueron menos afectados. Entre las zonas poco usuales se presentó un caso donde la lesión fue ubicada en la mama de una mujer y un paciente que presentó lesiones en dos regiones anatómicas distintas (brazo izquierdo y abdomen), probablemente 
debido a reinfección la que se presenta en individuos sensibilizados por falta de hábitos de higiene, por autoinoculación, rascado o infección por otras vías ${ }^{(18)}$.

El tiempo mínimo de lesión en nuestro estudio fue de una semana, lo cual coincide con los estudios realizados por García et al. ${ }^{(15)}$. El tiempo máximo fue de tres años. En 55 \% de los casos se observó que las lesiones presentaban menos de un mes de evolución. Esto puede deberse a que la mayoría de pacientes eran niños y acudían al centro médico por preocupación de sus padres, a diferencia de los adultos que suelen automedicarse o no prestar atención a la lesión.

El significado epidemiológico de los resultados hallados, comparando con los estudios de Abancay previos (3,8,9), muestra hallazgos similares en cuanto al grupo etario afectado, la predominancia del sexo masculino, y la presencia de lesiones en la cabeza y cuello. Nuestros hallazgos sugieren, en contraste con dichos estudios, la mayor predominancia de lesión cutánea fija. Sin embargo, el pequeño tamaño muestral limita las conclusiones de nuestro estudio. Pese a ello, el número de casos atendidos en un periodo corto sugiere que la esporotricosis sigue siendo un problema de salud pública en Abancay y, probablemente, en otras zonas agrícolas peruanas. Pese a que esta realidad se encuentra notificada desde hace una década, han sido pocas las medidas destinadas a su control. Abancay necesita la implementación de un programa de prevención y manejo de las labores domésticas para evitar la exposición al Sporothrix schenckii y para reducir el número de casos y, además, establecer técnicas de diagnóstico diferencial frente a otras infecciones que presentan manifestaciones clínicas similares a la esporotricosis.

En conclusión, la esporotricosis es una infección frecuente en la población de Abancay, referida al Centro Médico Santa Teresa y debe tenerse en cuenta no solo como enfermedad ocupacional sino, además, en población pediátrica. La esporotricosis debe incluirse en el diagnóstico diferencial no solo de lesiones en extremidades sino también en presencia de lesiones en cabeza y cuello, particularmente en pacientes provenientes de zonas endémicas.

\section{AGRADECIMIENTOS}

A la Blga. Carmen Motta Mariaca y al técnico Walter Zara por su colaboración en los procedimientos diagnósticos.

\section{Contribuciones de autoría}

MCRS, JAC y EGAA diseñaron el estudio; JLT, MCRS, JAC y JDPO participaron en la recolección de datos y procesamiento de las muestras biológicas; MCRS, JAC y EGAA realizaron el análisis estadístico. Todos los autores contribuyeron en la elaboración del manuscrito, revisión crítica y autorización de documento final.

\section{Fuentes de financiamiento}

El estudio contó con el apoyo técnico-financiero del Centro Médico Santa Teresa de Abancay y el Laboratorio referencial de salud pública de la DIRESA-Apurímac.

\section{Conflictos de interés}

Los autores declaran no tener conflictos de interés en la publicación de este artículo.

\section{REFERENCIAS BIBLIOGRÁFICAS}

1. Rippon J. Micología médica. Hongos y actinomicetos patógenos. $3^{a}$ ed. México: Editorial Interamericana; 1990.

2. De Lima M, De Oliveira A, Francesconi A, Gutierrez M, Conceicao F, Pacheco, et al. Cat-Transmitted Sporotrichosis Epidemic in Rio de Janeiro, Brazil. Description of a series of cases. Clin Infect Dis. 2004;38:529-35.

3. Flores A, Indacochea S, De La Fuente J, Bustamante B, Holgado W. Esporotricosis en Abancay, Perú. Rev Per Epid. 1991;4(1):5-10.

4. Arenas R. Atlas diagnóstico y tratamiento, micosis profundas. 2da ed. México: Mc. Graw-Hill, Interamericana; 1995. p. 346-58.

5. Arenas R. Esporotricosis en: Micología médica ilustrada. 2da ed. México: Mc Graw Hill Interamericana; 2003. p. 129-38.

6. Sánchez M, Araiza J, Bonifaz A. Aislamiento y caracterización de cepas silvestres de Sporotrhix schenkii e investigaciones de reactores a la esporotricina. Gac Med Mex. 2004;140(5):507-12.

7. García, R. Esporotricosis en a altura del Cusco-Perú. Nueva zona endémica. Experiencia de once años. Folia Dermatol Perú. 1998;9(1-2):59-61.

8. Lyon G, Zurita S, Casquero J, Holgado W, Guevara J, Brandt M, et al. Population-Based Surveillance and a Case-Control Study of Risk Factors for Endemic Lymphocutaneous Sporotrichosis in Perú. Clin infect Dis. 2003;36:349.

9. Pappas P, Tellez I, Deep A, Nolasco D, Holgado W, Bustamante B. Sporotrichosis in Perú: Description of an Area Hyperendemicity. Clin Infect Dis. 2000;30:65-70.

10. Gaitán I. Actividad de doce plantas nativas guatemaltecas contra Sporothrix schenckii [Tesis doctoral]. Guatemala: Universidad de San Carlos de Guatemala; 2005.

11. Méndez L, Anides A, Peña G, Manzano P, López R, Hernández $F$, et al. Esporotricosis cutánea fija incógnita. Rev Iberoam Micol. 2004;21:150-2.

12. Nobre M, De Castro A, Caetano D, De Souza L, Meireles M, Ferreiro L. Recurrencia de esporotricosis en gatos con implicancias zoonóticas. Rev Iberoam Micol. 2001;18:137-40.

13. Munguía R, Romo Y, Castañeda E, Velasquez M. Epidemiología de la esporotricosis en el municipio de Huauchinango, Puebla. Enf Inf Microbiol. 2007;27(2):38-43. 
14. Miranda H. Características clínicas e incidencia de la esporotricosis palpebral: comunicación de 16 casos nuevos. Folia Dermatol Peru. 2006;17(1):9-15.

15. García M, Urquiaga T, López N, Urquiaga J. Esporotricosis cutánea en niños en un Hospital Regional del Perú. Dermatol Peru. 2004;14(2):104-9.

16. Aguilar W. Detección de dermatofitos en los focos de Sporothrix schenckii H\&P en la ciudad de Abancay. [Tesis de bachiller]. Cusco: Facultad de Ciencias Biológicas, Universidad Nacional San Antonio Abad del Cusco; 1995.

17. Quiñones A, Alió A, Aranzazu N, Zerpa O, Cavallera E, Reyes O, et al. Esporotricosis: presentación inusual. Dermatología Venezolana. 1998;36(3):101-4
18. Padilla M, Siordia S, Novales J. Esporotricosis con involución espontánea. Dermatología Rev Mex. 2007;51:14-9.

Correspondencia: Max Carlos Ramírez Soto

Dirección: Universidad Nacional de San Antonio Abad del Cusco. Av. de la Cultura N 733. Cusco, Perú.

Teléfono: 984-887238

Correo electrónico: maxcrs22@gmail.com

\section{Suscríbase en forma electrónica y gratuita a los contenidos de la Revista Peruana de Medicina Experimental y Salud Pública, ingrese a WWW.ins.gob.pe, seleccione el ícono de la revista y envíenos sus datos.}

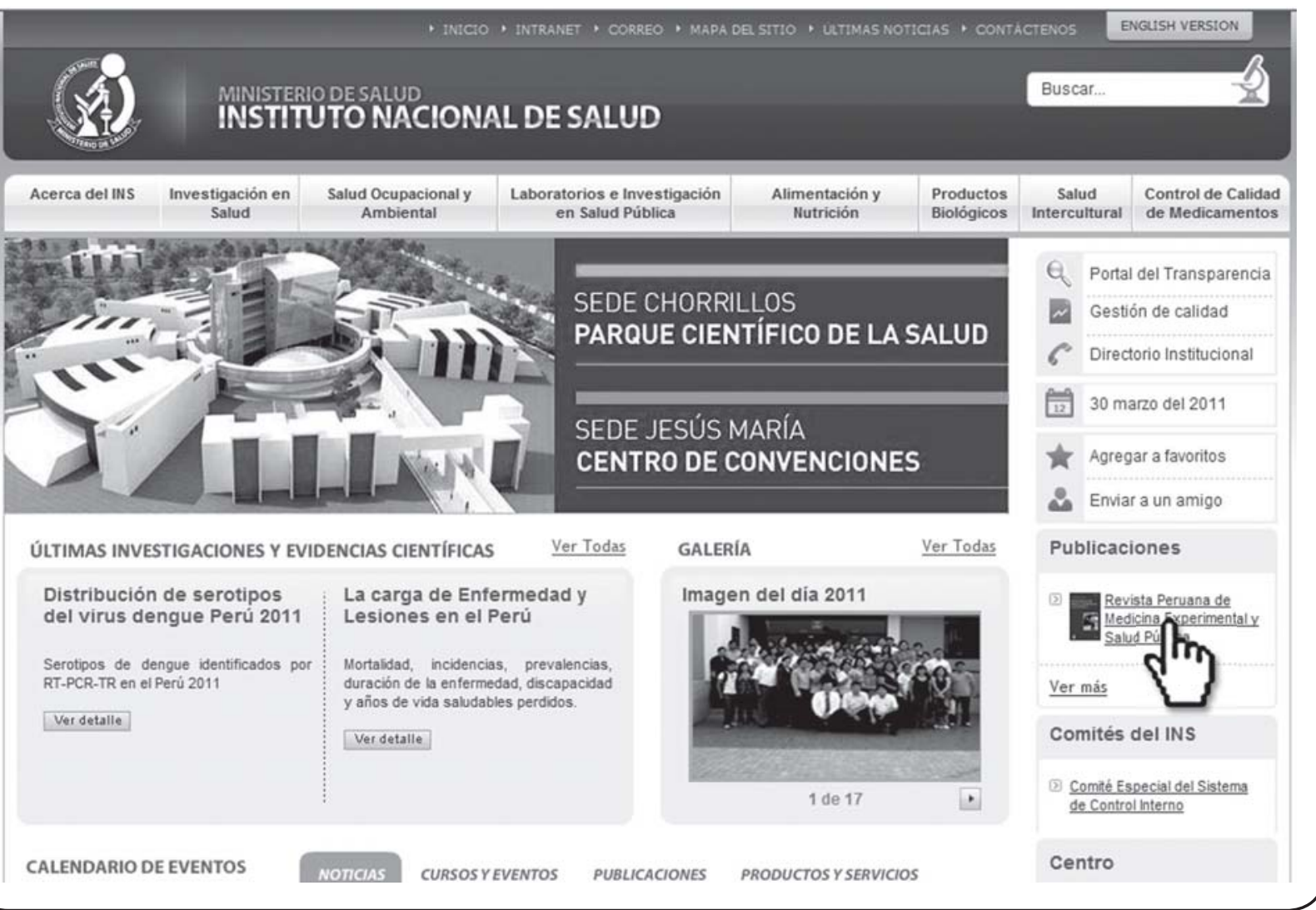

